\title{
La violencia escolar en los alumnos de la carrera de Medicina del Centro Interdisciplinario de Ciencias de la Salud, Unidad Milpa Alta, del Instituto Politécnico Nacional
}

\author{
School violence in the students of the Medical career of the Centro \\ Interdisciplinario de Ciencias de la Salud, Unidad Milpa Alta of the Instituto \\ Politécnico Nacional
}

Violência escolar nos discentes da carreira de medicina do Centro Interdisciplinar de Ciências da Saúde da Unidade Milpa Alta, do Instituto

Politécnico Nacional

Elias Fuentes Aguilar

Instituto Politécnico Nacional, México

efuentes73@hotmail.com

https://orcid.org/0000-0002-9348-9620

\section{Resumen}

La violencia escolar ha aumentado en los últimos años, lo cual es preocupante debido a que puede conducir a una violencia extrema. Los estudios realizados sobre este tema son generalmente aplicados en primarias y secundarias; hay pocos estudios a nivel medio y nivel superior. Este trabajo se realizó en una escuela de nivel superior del Instituto Politécnico Nacional; se aplicó a 350 alumnos de la carrera de Médico Cirujano y Partero, contemplando nueve preguntas modificadas del cuestionario de violencia escolar (Cuve). El cuestionario contempló los siguientes tipos de violencia: verbal de alumnos hacia alumnos, verbal del alumno hacia el profesor, disrupción en el aula, violencia física directa y amenazas entre estudiantes, violencia física indirecta por parte del alumnado, exclusión social, violencia a través de las tecnologías de la información y de la comunicación (TIC) y violencia del profesor hacia el alumno. De los resultados se puede mencionar que el rubro que despertó 
mayor interés fue el de la violencia del profesor hacia el alumno, por lo que se deben de plantear estrategias para disminuir la incidencia de este tipo de acciones.

Palabra claves: medicina, violencia escolar, violencia del profesor.

\section{Abstract}

School violence has increased in recent years, this is worrisome because it can lead to extreme violence, the studies carried out are generally in primary and secondary, there are few studies of the middle and upper level, this work was carried out in a Higher level school of the Instituto Politecnico Nacional, applied to 350 students of the career of Medicine, contemplating nine modified questions of the CUVE (school violence questionnaire), and were the following: Verbal violence of students towards students, Verbal violence of the student to the teacher, Disruption in the classroom, direct physical violence and threats among students, indirect physical violence by students, social exclusion, violence through information and communication technologies and teacher violence towards the student, of the results we can mention that the question that drew attention was the teacher's violence ia the student so they should consider strategies to reduce their incidence of this question.

Keywords: medicine, school violence, teacher violence.

\section{Resumo}

A violência escolar aumentou nos últimos anos, o que é preocupante porque pode levar a uma violência extrema. Os estudos realizados sobre este tema são geralmente aplicados em escolas primárias e secundárias; Existem poucos estudos nos níveis médio e superior. Este trabalho foi realizado em uma escola de nível superior do Instituto Politécnico Nacional; foi aplicado a 350 estudantes da carreira de Cirurgião e Obstetra, contemplando nove questões modificadas do questionário de violência escolar (Cuve). O questionário contemplou os seguintes tipos de violência: verbal de estudantes para estudantes, verbal do aluno para o professor, interrupção na sala de aula, violência física direta e ameaças entre os alunos, violência física indireta pelos estudantes, exclusão social, violência através de as tecnologias da informação e comunicação (TIC) e a violência do professor para com o aluno. A partir dos resultados, pode-se mencionar que o item que despertou maior interesse foi o da violência 
do professor para com o aluno, portanto estratégias devem ser propostas para reduzir a incidência desse tipo de ação.

Palavras-chave: medicina, violência escolar, violência de professores.

Fecha Recepción: Junio 2017 Fecha Aceptación: Enero 2020

\section{Introducción}

La violencia es una actitud o comportamiento que constituye una violación o un arrebato al ser humano de algo que le es esencial como persona (integridad física, psíquica, moral, derechos y libertades). También se puede definir como aquella conducta intencionada con la que se causa un daño o un perjuicio (Álvarez, Rodríguez, González, Núñez y Álvarez, 2010).

La violencia escolar es un fenómeno cada vez más frecuente entre los jóvenes de ambos sexos. El estudio de este generalmente se ha centrado en su presencia en planteles de secundaria y preparatoria, abarcando temas como el bullying y la violencia en el noviazgo. Sin embargo, a nivel superior no se está exento de padecer dicho problema. Esta cuestión involucra afectaciones tanto en lo físico, como en lo verbal o inclusive la exclusión social. Tiene factores internos y externos: entre los internos se encuentran los propios de la persona (personalidad y estado de ánimo, entre otros) y los factores externos se encuentran la violencia familiar, el estado socioeconómico, una familia disfuncional y los maestros.

Desde los años setenta, ha venido en aumento en varios países este problema. En Mexico se empezó a detectar, cada vez más, como consecuencia de la crisis social, cultural y familiar que se registra en la actualidad (Tapia, 2012).

Cabe mencionar que puede provenir de personas o instituciones y realizarse de forma pasiva o activa, ya que aparte de la violencia directa (golpes o destrucción visible) también se encuentran las formas indirectas o sutiles, como la violencia psicológica. Por eso es que estas actitudes se reflejan tanto en el alumno como en el docente (Adaszco y Kornblit, 2008). Si bien pueden llegar a ser difíciles de reconocer, son cotidianas en ámbitos concretos como la escuela, una manifestación compleja con abordajes múltiples (Prieto, 2005). Desafortunadamente son muchos los estudios publicados solo para el nivel primaria, secundaria o preparatoria, como ya se mencionó, mientras que a nivel superior son escasos los artículos referidos (Orozco, 2012). 
Lo que sí es un hecho es que la violencia escolar cada día va más en aumento, ya sea verbal, física o psicológica (Álvarez, 2013). Es importante contemplar o mencionar que en el estudio de la violencia no solo debe involucrarse a los alumnos, sino también a los docentes, pues estos son también un factor importante en el contexto educativo (Díaz Aguado y Martínez, 2013)

En relación a la violencia escolar a nivel superior, estudios como el de Romero y Plata (2015) hacen referencia a esta y la relacionan con el enfoque de la competencia que se desarrolla entre los alumnos y la búsqueda de una supremacía en relación a quién es mejor en el aula.

No está de más recalcar que un factor importante en el aula es el docente, quien, según el estudio de Cervantes, Villalobos y Sanches (2013), puede llegar a desarrollar actitudes como ignorar, menospreciar y discriminar al alumno, así como presenciar actos de acoso sexual y físico.

Por lo tanto, este trabajo se plantea abordar los siguientes cuestionamientos: ¿Existe violencia en alumnos de nivel superior de la carrera de medicina? Y si existe, ¿qué forma es la que predomina más? ¿Existe violencia entre alumnos y docentes?

\section{Materiales y métodos}

Esta investigación es cuantitativa, descriptiva y transversal. Se usaron cuestionarios aplicados al azar. Con algunas modificaciones, estos cuestionarios tomaron como base el cuestionario de violencia escolar (Cuve); constó de ocho temas divididos en varios ítems. Se aplicó en el periodo agosto-octubre de 2017 de forma anónima a una muestra de 350 alumnos de todos los semestres de la carrera de Medicina, de un total de 500 alumnos, aproximadamente, del Centro Interdisciplinario de Ciencias de la Salud, Unidad Milpa Alta, del Instituto Politécnico Nacional.

Los temas que abarcó el cuestionario fueron los siguientes:

- Violencia verbal de alumnos hacia alumnos.

- Violencia verbal del alumno hacia el profesor.

- Disrupción en el aula.

- Violencia física directa y amenazas entre estudiantes.

- Violencia física indirecta por parte del alumnado. 
- $\quad$ Exclusión social.

- Violencia a través de las tecnologías de la información y de la comunicación (TIC).

- Violencia del profesor hacia el alumno.

Cada ítem tenía cinco opciones: nunca, casi nunca, a veces, muchas veces y siempre. Los alumnos contestaron todos los ítems, verificando que no quedaran ninguno sin contestar.

Los resultados se tabularon en SSPS versión 23.

\section{Resultados}

La media de la edad de los alumnos encuestados fue de 20 años y participaron 62.8 $\%$ de mujeres y $37.2 \%$ de hombres.

Tabla 1. Frecuencia de edad de los participantes

\begin{tabular}{||l|l|l|}
\hline Edad & Frecuencia & Porcentaje \\
\hline 17 & 1 & .3 \\
\hline 18 & 51 & 14.5 \\
\hline 19 & 71 & 20.2 \\
\hline 20 & 105 & 29.8 \\
\hline 21 & 23 & 6.5 \\
\hline 22 & 53 & 15.1 \\
\hline 23 & 40 & 11.4 \\
\hline 24 & 7 & 2.0 \\
\hline 25 & 1 & .3 \\
\hline Total & 352 & 100 \\
\hline
\end{tabular}

Fuente: Elaboración propia

Tabla 2. Frecuencia de sexo por participantes

\begin{tabular}{||l|l|l|}
\hline Sexo & Frecuencia & Porcentaje \\
\hline Hombre & 131 & 37.2 \\
\hline Mujer & 221 & 62.8 \\
\hline Total & 352 & 100 \\
\hline
\end{tabular}

Fuente: Elaboración propia 


\section{Violencia verbal de alumnos hacia alumnos}

En este rubro se cuestionó a los participantes con los siguientes ítems:

- ¿Usted hace rumores negativos acerca de compañeros?

- ¿ ¿Usted pone apodo a sus compañeros?

- ¿ ¿Usted ha insultado a sus compañeros?

- ¿ ¿A usted le han puesto apodos?

- ¿A usted lo han insultado?

Tabla 3. Frecuencia de los rumores negativos realizados a algunos compañeros

\begin{tabular}{||l|l|l|}
\hline $\begin{array}{l}\text { ¿Usted hace rumores negativos acerca de } \\
\text { compañeros? }\end{array}$ & Frecuencia & Porcentaje \\
\hline Nunca & 258 & 73.3 \\
\hline Casi nunca & 62 & 17.6 \\
\hline A veces & 32 & 9.1 \\
\hline Total & 352 & 100 \\
\hline
\end{tabular}

Fuente: Elaboración propia

Se aprecia que $73 \%$ de los alumnos nunca han hecho rumores sobre sus demás compañeros. Sin embargo, $17 \%$ dice que casi nunca y solo $9 \%$ dice que a veces hace rumores negativos.

Tabla 4. Frecuencia sobre si los alumnos ponen apodos a otros alumnos

\begin{tabular}{||l|l|l|}
\hline ¿Usted pone apodos a sus compañeros? & Frecuencia & Porcentaje \\
\hline Nunca & 211 & 59.9 \\
\hline Casi nunca & 54 & 15.3 \\
\hline A veces & 75 & 21.3 \\
\hline Muchas veces & 6 & 1.7 \\
\hline Siempre & 6 & 1.7 \\
\hline Total & 352 & 100 \\
\hline
\end{tabular}

Fuente: Elaboración propia

Casi el $60 \%$ de los alumnos nunca ha puesto apodos a sus compañeros, en contraste con un $21 \%$ que menciona a veces suele poner apodos a sus compañeros. 
Tabla 5. Frecuencia sobre los alumnos que han insultado a compañeros

\begin{tabular}{||l|l|l|}
\hline ¿Usted ha insultado a sus compañeros? & Frecuencia & Porcentaje \\
\hline Nunca & 232 & 65.9 \\
\hline Casi nunca & 60 & 17.0 \\
\hline A veces & 55 & 15.6 \\
\hline Muchas veces & 4 & 1.1 \\
\hline Siempre & 1 & .3 \\
\hline Total & 352 & 100 \\
\hline
\end{tabular}

Fuente: Elaboración propia

Por su parte, $65.9 \%$ de los alumnos menciona que nunca han insultados a sus compañeros, un $17 \%$ comenta que casi nunca y $15 \%$ refiere que a veces.

Tabla 6. Frecuencia sobre los alumnos que les han puesto apodos

\begin{tabular}{||l|l|l|}
\hline iA usted le han puesto apodos? & Frecuencia & Porcentaje \\
\hline Nunca & 176 & 50.0 \\
\hline Casi nunca & 74 & 21.0 \\
\hline A veces & 73 & 20.7 \\
\hline Muchas veces & 29 & 8.2 \\
\hline Total & 352 & 100 \\
\hline
\end{tabular}

Fuente: Elaboración propia

La mitad de los alumnos encuestados menciona que nunca les han puesto apodos, 21 $\%$ refiere que casi nunca, $20 \%$ menciona que a veces y $8 \%$ lo refiere como muchas veces.

Tabla 7. Frecuencia sobre alumnos que los han insultado

\begin{tabular}{||l|l|l|}
\hline ¿A usted lo han insultado? & Frecuencia & Porcentaje \\
\hline Nunca & 163 & 46.3 \\
\hline Casi nunca & 86 & 24.4 \\
\hline A veces & 87 & 24.7 \\
\hline Muchas veces & 14 & 4.0 \\
\hline Siempre & 2 & .6 \\
\hline Total & 352 & 100 \\
\hline
\end{tabular}

Fuente: Elaboración propia

El $24 \%$ de los alumnos mencionan que casi nunca los han insultado, en igual porcentaje, $24 \%$, mencionan que a veces y $4 \%$ muchas veces. 


\section{Violencia verbal del alumno hacia el profesor}

Para este rubro, por su parte, se consideraron los siguientes ítems:

- ¿Usted falta al respeto a su profesor en el aula?

- ¿Usted ha insultado a su profesor?

Tabla 8. Frecuencia sobre la falta de respeto del alumno hacia el profesor

\begin{tabular}{||l|l|l|}
\hline $\begin{array}{l}\text { ¿Usted falta al respeto a su profesor en el } \\
\text { aula? }\end{array}$ & Frecuencia & Porcentaje \\
\hline Nunca & 326 & 92.6 \\
\hline Casi nunca & 26 & 7.4 \\
\hline Total & 352 & 100 \\
\hline
\end{tabular}

Fuente: Elaboración propia

Respecto a que los alumnos le hayan faltado el respeto a su profesor, $92 \%$ dijeron no haberlo hecho y solo $7 \%$ lo menciona afirmativamente como casi nunca.

Tabla 9. Frecuencia de la falta de respeto que tiene el alumno hacia el profesor

\begin{tabular}{||l|l|l|}
\hline ¿Usted ha insultado a su profesor? & Frecuencia & Porcentaje \\
\hline Nunca & 343 & 97.4 \\
\hline Casi nunca & 7 & 2.0 \\
\hline A veces & 2 & 0.6 \\
\hline Total & 352 & 100 \\
\hline
\end{tabular}

Fuente: Elaboración propia

Mientras que en relación con haber insultado a su profesor, $97.4 \%$ de los alumnos refiere que nunca lo ha hecho y solo $2 \%$ menciona que casi nunca.

\section{Disrupción en el aula}

Para el rubro con relación a las conductas disruptivas dentro del aula, se tomaron en cuenta los siguientes ítems:

- ¿Usted ha dificultado las explicaciones del profesor hablando durante la clase?

- ¿Usted ha dificultado las explicaciones del profesor con su comportamiento durante la clase (salir constantemente, mover bancas, entre otras)? 
Tabla 10. Frecuencia sobre el alumno dificultando las explicaciones del profesor en clase

\begin{tabular}{|l|l|l|}
\hline $\begin{array}{l}\text { ¿Usted ha dificultado las explicaciones del } \\
\text { profesor hablando durante la clase? }\end{array}$ & Frecuencia & Porcentaje \\
\hline Nunca & 343 & 97.4 \\
\hline Casi nunca & 9 & 2.6 \\
\hline Total & 352 & 100 \\
\hline
\end{tabular}

Fuente: Elaboración propia

Mientras que $97 \%$ de los alumnos refiere que nunca han hablado en clase, solo $2 \%$ refiere que sí.

Tabla 11. Frecuencia sobre el alumno que dificulta las explicaciones del profesor

\begin{tabular}{|l|l|l|}
\hline $\begin{array}{c}\text { ¿Usted ha dificultado las explicaciones del profesor } \\
\text { con su comportamiento durante la clase (salir } \\
\text { constantemente, mover bancas, entre otras)? }\end{array}$ & \multicolumn{1}{|c|}{ Frecuencia } & Porcentaje \\
\hline Nunca & 213 & 60.5 \\
\hline Casi nunca & 100 & 28.4 \\
\hline A veces & 39 & 11.1 \\
\hline Total & 352 & 100 \\
\hline
\end{tabular}

Fuente: Elaboración propia

Finalmente, $60 \%$ de los alumnos refiere que nunca ha dificultado la explicación de los profesores; por otra parte, $28 \%$ menciona que casi nunca y solo $11 \%$ que a veces.

\section{Violencia física directa y amenazas entre estudiantes}

Los ítems que se consideraron para este rubro fueron:

- ¿ ¿Usted ha golpeado a compañeros dentro de la escuela?

- ¿Usted ha protagonizado agresiones físicas en las cercanías de la escuela?

- ¿ ¿Usted ha amenazado a otros de palabra para meterles miedo u obligarles a hacer cosas?

- ¿Usted ha amenazan a otros con navajas u otros objetos para intimidarles u obligarles a algo?

- ¿A usted lo han golpeado en la escuela?

- ¿A usted lo han amenazado con palabras para hacer cosas en la escuela?

- ¿ ¿A usted lo han amenazado con navajas u otros objetos para hacer cosas en la escuela? 
Tabla 12. Frecuencia sobre los alumnos que golpean a otros alumnos

\begin{tabular}{|l|l|l|}
\hline $\begin{array}{l}\text { ¿Usted ha golpeado a compañeros dentro de la } \\
\text { escuela? }\end{array}$ & Frecuencia & Porcentaje \\
\hline Nunca & 264 & 75.0 \\
\hline Casi nunca & 81 & 23.0 \\
\hline A veces & 7 & 2.0 \\
\hline Total & 352 & 100 \\
\hline
\end{tabular}

Fuente: Elaboración propia

El $75 \%$ de los alumnos mencionan que nunca lo han hecho, $23 \%$ que casi nunca y 2 $\%$ menciona que a veces.

Tabla 13. Frecuencia sobre qué tanto el alumno ha protagonizado agresiones físicas cercanas en la escuela

\begin{tabular}{|l|l|l|}
\hline $\begin{array}{l}\text { ¿Usted ha protagonizado agresiones físicas en } \\
\text { las cercanías de la escuela? }\end{array}$ & Frecuencia & Porcentaje \\
\hline Nunca & 328 & 93.2 \\
\hline Casi nunca & 17 & 4.8 \\
\hline A veces & 7 & 2.0 \\
\hline Total & 352 & 100 \\
\hline
\end{tabular}

Fuente: Elaboración propia

En el rubro de agresiones físicas, un 93\% mencionan que nunca han protagonizado eso, un $4.8 \%$ menciona que casi nunca y un $2 \%$ menciona que a veces.

Tabla 14. Frecuencia sobre las amenazas de los alumnos a otros alumnos

\begin{tabular}{|l|l|l|}
\hline ¿Usted ha amenazado a otros de palabra para & & \\
meterles miedo u obligarles a hacer cosas? & Frecuencia & Porcentaje \\
\hline Nunca & 328 & 93.2 \\
\hline Casi nunca & 22 & 6.3 \\
\hline A veces & 2 & 6 \\
\hline Total & 352 & 100 \\
\hline
\end{tabular}

Fuente: Elaboración propia

Se aprecia que $93.2 \%$ de los alumnos dijeron que nunca han amenazado a sus compañeros para que hagan alguna cosa, $6.3 \%$ que casi nunca y el $0.6 \%$ que a veces. 
Tabla 15. Frecuencia sobre los alumnos que amenazan con objetos a otros alumnos

\begin{tabular}{||l|l|l|}
\hline $\begin{array}{l}\text { iUsted ha amenazado a otros con navajas u } \\
\text { otros objetos para intimidarles u obligarles a } \\
\text { algo? }\end{array}$ & Frecuencia & Porcentaje \\
\hline Nunca & 346 & 98.3 \\
\hline Casi nunca & 6 & 1.7 \\
\hline Total & 352 & 100 \\
\hline
\end{tabular}

Fuente: Elaboración propia

Si bien $98.3 \%$ de los alumnos menciona que nunca han amenazado a sus compañeros con objetos, $1.7 \%$ menciona que casi nunca.

Tabla 16. Frecuencia sobre los alumnos que han sido golpeados

\begin{tabular}{||l|l|l|}
\hline ¿A usted lo han golpeado en la escuela? & Frecuencia & Porcentaje \\
\hline Nunca & 345 & 98.0 \\
\hline Casi nunca & 7 & 2.0 \\
\hline Total & 352 & 100 \\
\hline
\end{tabular}

Fuente: Elaboración propia

En la tabla 16, se aprecia que $98 \%$ de los alumnos menciona que nunca los han golpeado, aunque $2 \%$ menciona que sí han sido golpeados.

Tabla 17. Frecuencia de alumnos que han sido amenazados con palabras

\begin{tabular}{||l|l|l|}
\hline $\begin{array}{l}\text { ¿A usted lo han amenazado con palabras para } \\
\text { hacer cosas en la escuela? }\end{array}$ & Frecuencia & Porcentaje \\
\hline Nunca & 348 & 98.9 \\
\hline casi nunca & 4 & 1.1 \\
\hline Total & 352 & 100 \\
\hline
\end{tabular}

Fuente: Elaboración propia

En este ítem (ver tabla 17), $98.9 \%$ de los alumnos mencionan que nunca los han amenazado para hacer cosas y solo $1.1 \%$ menciona que casi nunca. 
Tabla 18. Frecuencia de alumnos que han sido amenazados con objetos para hacer cosas en la escuela

\begin{tabular}{||l|l|l|}
\hline $\begin{array}{l}\text { ¿Usted lo han amenazado con navajas u otros } \\
\text { objetos para hacer cosas en la escuela? }\end{array}$ & Frecuencia & Porcentaje \\
\hline Nunca & 351 & 99.7 \\
\hline Casi nunca & 1 & 0.3 \\
\hline Total & 352 & 100 \\
\hline
\end{tabular}

Fuente: Elaboración propia

Sobre si el alumno recibió o no amenazas con objetos, $99.7 \%$ menciona que nunca y $0.3 \%$ menciona que casi nunca.

\section{Violencia física indirecta por parte del alumnado}

Los ítems a continuación enlistados fueron los que se tomaron en consideración para evaluar este tipo de violencia en un ambiente escolar:

- ¿Usted ha robado objetos o dinero en el centro educativo?

- ¿USted ha robado objetos o dinero de otros compañeros o compañeras?

- ¿Usted ha robado cosas de su profesor?

- ¿Usted ha escondido pertenencias o material del profesor para molestarlo intencionalmente?

- ¿Usted ha causado desperfectos intencionadamente en pertenencias del profesor?

Tabla 19. Frecuencia de alumnos que han hurtado en la escuela

\begin{tabular}{||l|l|l|}
\hline $\begin{array}{l}\text { ¿Usted ha robado objetos o dinero en el centro } \\
\text { educativo? }\end{array}$ & Frecuencia & Porcentaje \\
\hline Nunca & 350 & 99.4 \\
\hline Casi nunca & 1 & .3 \\
\hline A veces & 1 & .3 \\
\hline Total & 352 & 100 \\
\hline
\end{tabular}

Fuente: Elaboración propia

El $99.4 \%$ de los alumnos menciona que nunca han robado objetos o dinero en la escuela, $0.3 \%$ menciona que casi nunca y una igual proporción menciona que a veces. 
Tabla 20. Frecuencia sobre los alumnos que han hurtado a otros alumnos

\begin{tabular}{||l|l|l|}
\hline $\begin{array}{l}\text { ¿Usted ha robado objetos o dinero de otros } \\
\text { compañeros o compañeras? }\end{array}$ & Frecuencia & Porcentaje \\
\hline Nunca & 351 & 99.7 \\
\hline Casi nunca & 1 & .3 \\
\hline Total & 352 & 100 \\
\hline
\end{tabular}

Fuente: Elaboración propia

En la tabla 20 se observa que $99.7 \%$ de los alumnos dijeron nunca haber robado objetos o dinero y $0.3 \%$ menciona que casi nunca lo ha hecho.

Tabla 21. Frecuencia de alumnos que han robado cosas a su profesor

\begin{tabular}{||l|l|l|}
\hline ¿Usted ha robado cosas de su profesor? & Frecuencia & Porcentaje \\
\hline Nunca & 351 & 99.7 \\
\hline Casi nunca & 1 & .3 \\
\hline Total & 352 & 100 \\
\hline
\end{tabular}

Fuente: Elaboración propia

Casi la totalidad de alumnos nunca han robado cosas a su profesor, $99.7 \%$; solo $0.3 \%$ mencionó que casi nunca.

Tabla 22. Frecuencia de alumnos que esconden pertenencias a su profesor

\begin{tabular}{|l|l|l|}
\hline $\begin{array}{l}\text { USted ha escondido pertenencias o material } \\
\text { del profesor para molestarlo intencionalmente? }\end{array}$ & Frecuencia & Porcentaje \\
\hline Nunca & 352 & 100 \\
\hline
\end{tabular}

Fuente: Elaboración propia

El total de los alumnos mencionan que nunca han escondido pertenencias.

Tabla 23. Frecuencia de alumnos que han causado desperfectos en pertenencias de los profesores

\begin{tabular}{||l|l|l|}
\hline $\begin{array}{l}\text { ¿Usted ha causado desperfectos intencionadamente } \\
\text { en pertenencias del profesor? }\end{array}$ & Frecuencia & Porcentaje \\
\hline Nunca & 338 & 96.0 \\
\hline Casi nunca & 14 & 4.0 \\
\hline Total & 352 & 100.0 \\
\hline
\end{tabular}

Fuente: Elaboración propia

El 96\% de los alumnos menciona que nunca han causado un desperfecto intencional y solo el $4 \%$ menciona que casi nunca. 


\section{Exclusión social}

En este apartado los siguientes ítems fueron abordados:

- ¿Usted ha sido discriminado por sus compañeros por diferencias culturales, étnicas, religiosas o de género?

- ¿ ¿Usted ha sido discriminado por sus compañeros por bajas notas?

- $\quad$ ¿Usted ha sido discriminado por sus compañeros por sus buenos resultados académicos?

- ¿Usted ha discriminado a compañeros por diferencias culturales, étnicas, religiosas, etcétera?

- ¿Usted ha discriminado a compañeros por bajas notas?

- ¿ ¿Usted ha discriminado a compañeros por buenos resultados académicos?

Tabla 24. Frecuencia de alumnos que han sido discriminados por ideologías diversas

\begin{tabular}{|l|l|l|}
\hline $\begin{array}{c}\text { ¿Usted ha sido discriminado por sus } \\
\text { compañeros por diferencias culturales, étnicas, } \\
\text { religiosas o de género? }\end{array}$ & Frecuencia & Porcentaje \\
\hline Nunca & 302 & 85.8 \\
\hline Casi nunca & 36 & 10.2 \\
\hline A veces & 14 & 4.0 \\
\hline Total & 352 & 100 \\
\hline
\end{tabular}

Fuente: Elaboración propia

El $85.8 \%$ de los alumnos menciona que nunca han sido discriminados por diferencias culturales o religiosas, un $10.2 \%$ casi nunca y un $4 \%$ a veces.

Tabla 25. Frecuencia de alumnos que han sido discriminados por bajas notas

\begin{tabular}{|l|l|l|}
\hline $\begin{array}{l}\text { ¿Usted ha sido discriminado por sus compañeros } \\
\text { por bajas notas? }\end{array}$ & Frecuencia & Porcentaje \\
\hline Nunca & 342 & 97.2 \\
\hline Casi nunca & 9 & 2.6 \\
\hline A veces & 1 & .3 \\
\hline Total & 352 & 100 \\
\hline
\end{tabular}

Fuente: Elaboración propia

El $97.2 \%$ de los alumnos mencionan que nunca han sido discriminados por sacar bajas notas, $2.6 \%$ menciona que casi nunca y solo $0.3 \%$ menciona que a veces. 
Tabla 26. Frecuencia de alumnos que han sido discriminados por buenas notas

\begin{tabular}{||l|l|l|}
\hline $\begin{array}{l}\text { ¿Usted ha sido discriminado por sus compañeros } \\
\text { por sus buenos resultados académicos? }\end{array}$ & Frecuencia & Porcentaje \\
\hline Nunca & 312 & 88.6 \\
\hline casi nunca & 36 & 10.2 \\
\hline a veces & 4 & 1.1 \\
\hline Total & 352 & 100 \\
\hline
\end{tabular}

Fuente: Elaboración propia

El $88.6 \%$ de los alumnos menciona que nunca han sido discriminados por sus buenos resultados académicos, $10 \%$ menciona que casi nunca y solo $1.1 \%$ menciona que a veces.

Tabla 27. Frecuencia de alumnos que han sido discriminados por diferencias culturales

\begin{tabular}{||l|l|l|}
\hline $\begin{array}{l}\text { ¿Usted ha discriminado a compañeros por } \\
\text { diferencias culturales, étnicas o religiosas? }\end{array}$ & Frecuencia & Porcentaje \\
\hline Nunca & 312 & 88.6 \\
\hline Casi nunca & 28 & 8.0 \\
\hline A veces & 12 & 3.4 \\
\hline Total & 352 & 100 \\
\hline
\end{tabular}

Fuente: Elaboración propia

Se aprecia que $88.6 \%$ de los alumnos refiere nunca ha discriminado a sus compañeros por diferencias culturales, $8 \%$ refiere que casi nunca y $3.4 \%$ menciona que a veces.

Tabla 28. Frecuencia de alumnos discriminados por sacar bajas notas

\begin{tabular}{||l|l|l|}
\hline $\begin{array}{l}\text { ¿Usted ha discriminado a compañeros por bajas } \\
\text { notas? }\end{array}$ & Frecuencia & Porcentaje \\
\hline Nunca & 317 & 90.1 \\
\hline casi nunca & 34 & 9.7 \\
\hline a veces & 1 & .3 \\
\hline Total & 352 & 100.0 \\
\hline
\end{tabular}

Fuente: Elaboración propia

Asimismo, $90 \%$ de los alumnos refiere que nunca ha discriminado a sus compañeros por bajas notas, $9.7 \%$ casi nunca y $3 \%$ a veces. 
Tabla 29. Frecuencia de alumnos discriminados por buenas notas

\begin{tabular}{||l|l|l|}
\hline $\begin{array}{l}\text { ¿Usted ha discriminado a compañeros por buenos } \\
\text { resultados académicos? }\end{array}$ & Frecuencia & Porcentaje \\
\hline Nunca & 342 & 97.2 \\
\hline Casi nunca & 9 & 2.6 \\
\hline A veces & 1 & .3 \\
\hline Total & 352 & 100 \\
\hline
\end{tabular}

Fuente: Elaboración propia

Y finalmente, $97.2 \%$ de los alumnos menciona que nunca ha discriminado a sus compañeros por buenos resultados, $2.6 \%$ que casi nunca y solo $3 \%$ que a veces.

\section{Violencia a través de las tecnologías de la información y de la comunicación}

Los ítems que se consideraron para evaluar la violencia realizada a través de las TIC fueron los siguientes:

- ¿Usted ha publicado en Twitter, Facebook, etc., ofensas, insultos o amenazas al profesor?

- ¿Usted ha publicado en Internet fotos o vídeos ofensivos de profesores?

- ¿Usted ha publicado en Internet fotos o vídeos ofensivos de compañeros?

- ¿Usted envía a compañeros mensajes con el celular de ofensa, insulto o amenaza?

- ¿Usted ha grabado o fotografiado a compañeros para burlarse?

- ¿Ha recibido mensajes o insultos a través de Twitter, Facebook, etc.?

Tabla 30. Frecuencia de alumnos que publican en redes insultos o amenazas al profesor

\begin{tabular}{|l|l|l|}
\hline $\begin{array}{l}\text { ¿Usted ha publicado en Twitter, Facebook, } \\
\text { etc. ofensas, insultos o amenazas al } \\
\text { profesor? }\end{array}$ & Frecuencia & Porcentaje \\
\hline Nunca & 336 & 95.5 \\
\hline Casi nunca & 8 & 2.3 \\
\hline A veces & 8 & 2.3 \\
\hline Total & 352 & 100 \\
\hline
\end{tabular}

Fuente: Elaboración propia

El $95.5 \%$ de los alumnos refiere que nunca han publicado por este medio ofensas, y en igual proporción casi nunca y a veces $(2.3 \%)$. 
Tabla 31. Frecuencia de alumnos que publican en Internet fotos o videos de profesores

\begin{tabular}{||l|l|l|}
\hline $\begin{array}{l}\text { ¿Usted ha publicado en Internet fotos o vídeos } \\
\text { ofensivos de profesores? }\end{array}$ & Frecuencia & Porcentaje \\
\hline Nunca & 351 & 99.7 \\
\hline Casi nunca & 1 & .3 \\
\hline Total & 352 & 100 \\
\hline
\end{tabular}

Fuente: Elaboración propia

Solo el $0.3 \%$ refiere que casi nunca ha publicado fotos ofensivas de profesores y el resto que nunca.

Tabla 32. Frecuencia de alumnos que publican videos o fotos de compañeros

\begin{tabular}{|l|l|l|}
\hline $\begin{array}{l}\text { ¿Usted ha publicado en Internet fotos o vídeos } \\
\text { ofensivos de compañeros? }\end{array}$ & Frecuencia & Porcentaje \\
\hline Nunca & 338 & 96.0 \\
\hline Casi nunca & 14 & 4.0 \\
\hline Total & 352 & 100 \\
\hline
\end{tabular}

Fuente: Elaboración propia

Del total, $96 \%$ menciona que nunca han publicado fotos o videos ofensivos de sus compañeros y $4 \%$ que casi nunca.

Tabla 33. Frecuencia de alumnos que mandan mensajes ofensivos al celular a otros compañeros

\begin{tabular}{||l|l|l|}
\hline $\begin{array}{l}\text { ¿Usted envía a compañeros mensajes con el } \\
\text { celular de ofensa, insulto o amenaza? }\end{array}$ & Frecuencia & Porcentaje \\
\hline Nunca & 339 & 96.3 \\
\hline Casi nunca & 12 & 3.4 \\
\hline A veces & 1 & .3 \\
\hline Total & 352 & 100 \\
\hline
\end{tabular}

Fuente: Elaboración propia

Sobre la pregunta de que si los alumnos envían mensajes agresivos al celular, $96.3 \%$ menciona que nunca, $3.4 \%$ refiere que casi nunca y $0.3 \%$ refiere que a veces. 
Tabla 34. Frecuencia de alumnos que fotografían o graban a compañeros para burlarse.

\begin{tabular}{||l|l|l|}
\hline $\begin{array}{l}\text { ¿Usted ha grabado o fotografiado a compañeros, } \\
\text { para burlarse? }\end{array}$ & Frecuencia & Porcentaje \\
\hline Nunca & 323 & 91.8 \\
\hline Casi nunca & 15 & 4.3 \\
\hline A veces & 14 & 4.0 \\
\hline Total & 352 & 100.0 \\
\hline
\end{tabular}

Fuente: Elaboración propia

Sobre si los alumnos han fotografiado o grabado a otros compañeros para burlarse, $91.8 \%$ menciona que nunca, $4.3 \%$ que casi nunca y $4 \%$ que a veces.

Tabla 35. Frecuencia de alumnos que recibieron mensajes ofensivos a través de las redes sociales

\begin{tabular}{|l|l|l|}
\hline $\begin{array}{l}\text { ¿Ha recibido mensajes o insultos a través de } \\
\text { Twitter, Facebook, etc.? }\end{array}$ & Frecuencia & Porcentaje \\
\hline Nunca & 302 & 85.8 \\
\hline Casi nunca & 26 & 7.4 \\
\hline A veces & 24 & 6.8 \\
\hline Total & 352 & 100.0 \\
\hline
\end{tabular}

Fuente: Elaboración propia

La mayoría de los alumnos mencionan que nunca han recibido insultos a través de redes sociales $(85.8 \%)$, tan solo $7.4 \%$ refiere que casi nunca y solo $6.8 \%$ que a veces.

\section{Violencia del profesor hacia el alumno}

Para esta categoría se abordaron los siguientes cuestionamientos:

- ¿Usted cree que existan profesores que tienen preferencias por ciertos alumnos?

- ¿ ¿Usted cree que existan profesores que castigan injustamente?

- ¿Usted cree que existan profesores que ignoran a ciertos alumnos?

- ¿Usted cree que existan profesores que no escuchan a sus alumnos?

- ¿ ¿Usted cree que existan profesores que insulten a los alumnos?

- ¿ ¿Usted cree que el profesor baje calificaciones a alumnos sin justificación?

- ¿Usted cree que ciertos profesores intimiden o atemoricen a los alumnos?

- ¿ ¿Ha sido amenazado por algún docente? 
Tabla 36. Frecuencia de alumnos que creen que el profesor tiene preferencia por ciertos alumnos

\begin{tabular}{||l|l|l|}
\hline $\begin{array}{l}\text { ¿Usted cree que existan profesores que tienen } \\
\text { preferencias por ciertos alumnos? }\end{array}$ & Frecuencia & Porcentaje \\
\hline Nunca & 76 & 21.6 \\
\hline Casi nunca & 41 & 11.6 \\
\hline A veces & 131 & 37.2 \\
\hline Muchas veces & 74 & 21.0 \\
\hline Siempre & 30 & 8.5 \\
\hline Total & 352 & 100 \\
\hline
\end{tabular}

Fuente: Elaboración propia

El $21 \%$ de los alumnos refieren que no creen que exista profesores con preferencia, $11.6 \%$ refiere que casi nunca, $37.2 \%$ a veces, $21 \%$ muchas veces y $8.5 \%$ que siempre.

Tabla 37. Frecuencia de alumnos que creen que el profesor castiga injustamente

\begin{tabular}{|l|l|l|}
\hline $\begin{array}{l}\text { ¿Usted cree que existan profesores que } \\
\text { castigan injustamente? }\end{array}$ & Frecuencia & Porcentaje \\
\hline Nunca & 94 & 26.7 \\
\hline Casi nunca & 109 & 31.0 \\
\hline A veces & 119 & 33.8 \\
\hline Muchas veces & 24 & 6.8 \\
\hline Siempre & 6 & 1.7 \\
\hline Total & 352 & 100 \\
\hline
\end{tabular}

Fuente: Elaboración propia

El $26.7 \%$ de los alumnos refiere que nunca, $31 \%$ refiere que casi nunca, $33.8 \%$ a veces, $6.8 \%$ muchas veces y solo $1.7 \%$ refiere que siempre.

Tabla 38. Frecuencia de alumnos que creen que su profesor los ignora

\begin{tabular}{|l|l|l|}
\hline $\begin{array}{l}\text { iUsted cree que existan profesores que } \\
\text { ignoran a ciertos alumnos? }\end{array}$ & Frecuencia & Porcentaje \\
\hline Nunca & 85 & 24.1 \\
\hline Casi nunca & 107 & 30.4 \\
\hline A veces & 132 & 37.5 \\
\hline Muchas veces & 28 & 8.0 \\
\hline Total & 352 & 100 \\
\hline
\end{tabular}

Fuente: Elaboración propia

Del total, $24.1 \%$ de los alumnos no creen que existan profesores que ignoran a ciertos alumnos, $30 \%$ de los alumnos refiere que casi nunca, y $8 \%$ que muchas veces. 
Tabla 39. Frecuencia de alumnos que creen que el profesor no los escucha

\begin{tabular}{|l|l|l|}
\hline $\begin{array}{l}\text { ¿Usted cree que existan profesores que no } \\
\text { escuchan a sus alumnos? }\end{array}$ & Frecuencia & Porcentaje \\
\hline Nunca & 87 & 24.7 \\
\hline Casi nunca & 132 & 37.5 \\
\hline A veces & 93 & 26.4 \\
\hline Muchas veces & 40 & 11.4 \\
\hline Total & 352 & 100 \\
\hline
\end{tabular}

Fuente: Elaboración propia

Se observa que $24.7 \%$ de los alumnos dicen que no los escuchan, $37.5 \%$ refiere que casi nunca, $26.4 \%$ que a veces y $11.4 \%$ menciona que muchas veces.

Tabla 40. Frecuencia de alumnos que piensan que los profesores los insultan

\begin{tabular}{|l|l|l|}
\hline $\begin{array}{l}\text { ¿Usted cree que existan profesores que insulten } \\
\text { a los alumnos? }\end{array}$ & Frecuencia & Porcentaje \\
\hline Nunca & 158 & 44.9 \\
\hline Casi nunca & 120 & 34.1 \\
\hline A veces & 60 & 17.0 \\
\hline Muchas veces & 14 & 4.0 \\
\hline Total & 352 & 100 \\
\hline
\end{tabular}

Fuente: Elaboración propia

El $44.9 \%$ de los encuestados refiere que no creen que existan tales maestros, $34.1 \%$ refieren que casi nunca, $17 \%$ que a veces y $4 \%$ menciona que muchas veces.

Tabla 41. Frecuencia de alumnos que creen que el profesor baja calificaciones injustamente

\begin{tabular}{||l|l|l|}
\hline $\begin{array}{l}\text { ¿Usted cree que el profesor baje calificaciones a } \\
\text { alumnos sin justificación? }\end{array}$ & Frecuencia & Porcentaje \\
\hline Nunca & 121 & 34.4 \\
\hline Casi nunca & 147 & 41.8 \\
\hline A veces & 65 & 18.5 \\
\hline Muchas veces & 19 & 5.4 \\
\hline Total & 352 & 100 \\
\hline
\end{tabular}

Fuente: Elaboración propia

El $34.4 \%$ de los alumnos, refiere que no cree que pase algo así, $41.8 \%$ refiere que casi nunca, $18.5 \%$ refiere que a veces y $5.4 \%$ menciona que muchas veces. 
Tabla 42. Frecuencia de alumnos que creen que algunos profesores los intimidan

\begin{tabular}{||l|l|l|}
\hline $\begin{array}{l}\text { ¿Usted cree que ciertos profesores intimiden o } \\
\text { atemoricen a los alumnos? }\end{array}$ & Frecuencia & Porcentaje \\
\hline Nunca & 181 & 51.4 \\
\hline Casi nunca & 129 & 36.6 \\
\hline A veces & 30 & 8.5 \\
\hline Muchas veces & 12 & 3.4 \\
\hline Total & 352 & 100.0 \\
\hline
\end{tabular}

Fuente: Elaboración propia

La mitad de los estudiantes $(51.4 \%)$ menciona que nunca fueron víctimas de intimidación por parte del profesorado, y $3.4 \%$ mencionan que muchas veces.

Tabla 43. Frecuencia de alumnos que consideran que han sido amenazados por algún profesor

\begin{tabular}{||l|l|l|}
\hline ¿Ha sido amenazado por algún docente? & Frecuencia & Porcentaje \\
\hline Nunca & 298 & 84.7 \\
\hline Casi nunca & 42 & 11.9 \\
\hline A veces & 6 & 1.7 \\
\hline Muchas veces & 6 & 1.7 \\
\hline Total & 352 & 100 \\
\hline
\end{tabular}

Fuente: Elaboración propia

El $84.7 \%$ de los alumnos mencionan que nunca, $11.9 \%$ refieren que casi nunca y $a$ veces y muchas veces registra el mismo porcentaje (1.7\%).

\section{Discusión}

En el presente trabajo ningún rubro sale con porcentaje de $0 \%$, lo que indica que existe violencia, muchas veces disimulada, muchas veces tolerada, este estudio no se había realizado en ninguna escuela de nivel superior del instituto politécnico nacional del área medico biológica, los rubros que se plantean abarcan tanto del alumno - maestro, en todos los casos nos indican la violencia que existe en menor o mayor grado en la escuela, desafortunadamente no podemos hacer comparaciones con otros estudios de la carrea de medico cirujano y partero por que no lo hay.

Se considera que debe de haber mas estudios de este tipo, ya que un trabajador de la salud, llamese medico, odontólogo, enfermera etc. Debe desarrollarse en un ámbito sin violencia, ya que al final esto repercute en el paciente. 


\section{Conclusiones}

La violencia escolar es un problema en la mayoría de las escuelas de México ela cual se ha estado incrementando en las últimas décadas. Este estudio demuestra que el problema no solo radica entre alumnos, sino que también recae en la relación con los docentes, circunstancias que muchas veces no son contempladas.

Así, en este estudio, el alumno ve al docente como un elemento de poder. En los ítems que refieren a la violencia del docente hacia el alumno, el rubro de a veces podría ser un factor preocupante, aunque sin llegar al extremo de violencia física.

La cuestión, en este caso, es más compleja. Debido a que también debemos involucrar al docente en esta problemática, cosa que a veces omitimos o consideramos que el docente no es parte fundamental del problema, sin embargo esto es una cadena que si uno falla todos fallan, por lo que es importante que se impartan cursos para que esté preparado el docente en los conflictos entre alumnos y para que el mismo no caiga en la violencia hacia el alumnado.

\section{Referencias}

Adaszco, D. y Kornblit, A. (2008). Clima social escolar y violencia entre alumnos: Un vínculo explicativo posible. Un estudio en escuelas medias argentinas. En Berger, C. y Lisboa, C. (eds.), Violencia Escolar, estudios y posibilidades de intervención en Latinoamérica (pp. 109-138). Santiago de Chile, Chile: Editorial Universitaria.

Álvarez, D., González, P. y Álvarez, L. (2010). La formación de los futuros docentes frente a la violencia escolar. Revista de Psicodidactica, 15(1), 35-56.

Álvarez, D., Rodríguez, C., González, P., Núñez, J. C. y Álvarez, L. (2010). La formación de los futuros docentes frente a la violencia escolar. Revista de Psicodidáctica, 15(1), 35-56.

Álvarez, D., Núñez, J. y Dobarro, C. A. (2013). Cuestionarios para evaluar la violencia escolar en Educación Primaria y en Educación Secundaria: CUVE3-EP y CUVE3ESO. Apuntes de Psicología, 2(31), 191-202.

Cervantes M., Sánchez, C. y Villalobos, M. (2003). Percepción de la violencia del docente hacia el alumno en instituciones de educación superior. Trabajo presentado en el Primer Congreso Internacional de Investigación Educativa RIE-UANL. Nuevo León, del 28 al 30 de agosto de 2013. 
Díaz, M. J. and Martínez, R. (2013). Peer bullying and disruption coercion escalations in student-teacher relationship. Psicothema. 25(2), 206-213.

Debarbieux, E. (1996). International Survey of School Secundary School Students Questionnaire. Burdeos, Francia: Observatorio Europeo de la Violencia Escolar.

Orozco, L. A., Ybarra, J. L. y Guerra López, V. (2012). Adaptación del cuestionario de Violencia Escolar en estudiantes de educación secundaria de México. Revista mexicana de investigación en psicología, 1(4).

Tapia, D. (2012). La violencia escolar en las escuelas secundarias de México. (tesis licenciatura). Recuperada de https://www.uv.mx/iihs/files/2015/05/La-violenciaescolar-en-las-escuelas-secundarias-de-Mexico.pdf.

Prieto, M. T., Carrillo Navarro, J. C. y Jiménez Mora, J. (2005). La violencia escolar. Un estudio en el nivel medio superior. Revista Mexicana de Investigación Educativa, 27(10), 1027-1045.

Romero, A. y Plata, J. V. (2015). Acoso escolar en universidades. Enseñanza e Investigación en Psicología, 3(20), 266-274. 